\title{
Matching of Silicon Thin-Film Tandem Solar Cells for Maximum Power Output
}

\author{
C. Ulbrich, C. Zahren, A. Gerber, B. Blank, T. Merdzhanova, A. Gordijn, and U. Rau \\ Forschungszentrum Jülich, IEK5-Photovoltaik, 52425 Jülich, Germany \\ Correspondence should be addressed to C. Ulbrich; c.ulbrich@fz-juelich.de
}

Received 5 September 2012; Accepted 3 December 2012

Academic Editor: Franca Morazzoni

Copyright (C) 2013 C. Ulbrich et al. This is an open access article distributed under the Creative Commons Attribution License, which permits unrestricted use, distribution, and reproduction in any medium, provided the original work is properly cited.

\begin{abstract}
We present a meaningful characterization method for tandem solar cells. The experimental method allows for optimizing the output power instead of the current. Furthermore, it enables the extraction of the approximate AM1.5g efficiency when working with noncalibrated spectra. Current matching of tandem solar cells under short-circuit condition maximizes the output current but is disadvantageous for the overall fill factor and as a consequence does not imply an optimization of the output power of the device. We apply the matching condition to the maximum power output; that is, a stack of solar cells is power matched if the power output of each subcell is maximal at equal subcell currents. The new measurement procedure uses additional light-emitting diodes as bias light in the $J V$ characterization of tandem solar cells. Using a characterized reference tandem solar cell, such as a hydrogenated amorphous/microcrystalline silicon tandem, it is possible to extract the AM1.5g efficiency from tandems of the same technology also under noncalibrated spectra.
\end{abstract}

\section{Introduction}

The electrical series connection in tandem solar cells requires a design of the electrical and optical properties of each subcell that minimizes the losses arising from an electrical mismatch. Mismatching losses are eclipsed if each subcell delivers its maximum power output at the same current. Unfortunately, this power matching condition is difficult to access experimentally. Usually, the matching (or mismatch) of tandem stacks is investigated in terms of the current matching condition [1], which is achieved if the short-circuit currents of the subcells are equal. This condition is accessible by investigation of the spectral response or quantum efficiency of the subcells.

However, current and power matching conditions may differ significantly especially if the fill factors of the subcells are markedly different, like in hydrogenated amorphous/microcrystalline silicon (a-Si:H/ $\mu \mathrm{c}-\mathrm{Si}: \mathrm{H})$ thin-film tandem cells. This fact is illustrated by Figure 1 displaying simulated power density versus current density curves for two different a-Si:H/ $\mu \mathrm{c}-\mathrm{Si}: \mathrm{H}$ tandem stacks. The gray lines correspond to the respective subcell and overall $P J$ curves of a stack under current matching condition where the current density at zero voltage is maximized for the superposed $P J$ curve. The black lines correspond to a powermatched tandem cell. At the maximum power point, the power of the current-matched cell is outperformed by the power-matched tandem cell. Power matching implies a loss in total short-circuit current, that is, however, overcompensated at the maximum power point by the advantageous shape (advantageous overall fill factor) of the total $P J$ characteristics.

It is not possible to probe experimentally the single cell components of a tandem cell independently, neither electrically nor optically. To reveal some information on the single cell components of a tandem stack, an experimental method has been proposed that consists of recording the current density voltage- $(J V)$-characteristic of a sample using additional light sources. The method uses an approach similar to the light biasing in quantum efficiency measurement, however, in contrast to the wavelength resolved quantum efficiency measurement it is capable of determining the solar cell power under the changed illumination conditions, whereas the quantum efficiency measurement gives only information on the current matching. 


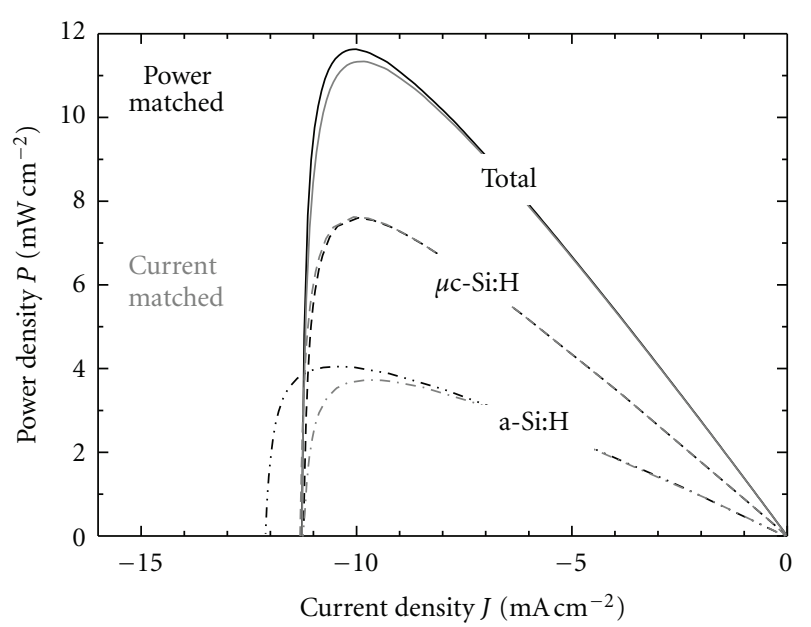

FIGURE 1: Simulated current-power characteristics of the subcells (dash-dotted: top cell, dashed line: bottom cell) and of the combined hydrogenated amorphous/microcrystalline tandem solar cell (straight line). The output power is maximized to $11.6 \mathrm{mWcm}^{-2}$ instead of $11.3 \mathrm{mWcm}^{-2}$, corresponding to a gain of $2.6 \%$ when the currents of the subcells are power matched; that is, the currents are matched at the maximum power point (black) instead of the common current matching at zero voltage (gray). The total thickness of the simulated tandems was $1.5 \mu \mathrm{m}$.

The method was proposed by Burdick and Glatfelter in 1986 [2]. The additional bias light is chosen to affect mainly either the top or the bottom cell of the tandem. The supplementary bias light increases the fraction of absorbed illumination in the respective subcell similar to an increased thickness of the absorber layer.

"Spectrometric characterization" methods have been applied to various types of solar cells, for example, crystalline (with a light-emitting diode (LED) solar simulator [3]), multijunction (with filtered and intensity-adapted sun simulator light $[4,5]$ ), and concentrator cells (adjusting the intensity of independent light sources [6] or using filters for a single-source sun simulator [7]). Burdick and Glatfelter used a high-intensity filament lamp, a Xenon-arc lamp, and a monochromator. LEDs offer more opportunities to easily shape further interesting experiments (see, e.g., [3]).

As the amorphous top layer of thin-film silicon tandem solar cells is known to degrade under light soaking, the optimization of tandem solar cells is specifically difficult and time consuming. The correlation of matching conditions and degradation has already been investigated by Repmann et al. [8], addressing also the effect on outdoor yield. The experimental study was, however, based on a series of tandem solar cells with various $i$-layer thicknesses as well as modeling and did not use the characterization method presented in this paper. The method is very helpful in such an analysis. The use of the method in the optimization of tandem solar cells minimizes the need to fabricate solar cells of different thicknesses.

In this paper, we present an experimental method based on the use of a white light source supplemented by additional blue and infrared LED arrays. This method allows for the

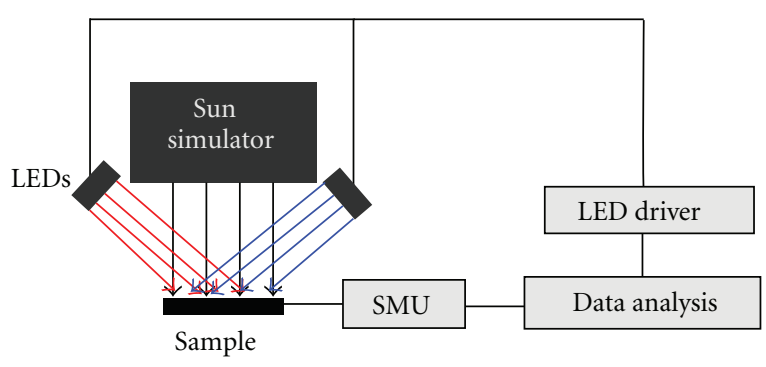

FIGURE 2: The experimental setup consists of a sun simulator that illuminates the solar cell. Additional illumination comes from two LED sources emitting at $\sim 450 \mathrm{~nm}$ and $850 \mathrm{~nm}$.

determination of the power mismatch of tandem solar cells, that is, the required change in layer thickness to reach maximum power. We refer to matching with respect to a maximum overall power instead of a maximum current under short-circuit conditions. Power matching is achieved for a stack of solar cells if the power output of each cell is maximal at equal cell currents. The experimental method serves to find the needed absorber layer thicknesses by changing the spectrum that can be made equivalent to changing the layer thickness and, thus, slightly shifting the single-cell $J V$ curves. We demonstrate the method using a reference tandem solar cell that is bottom limited in terms of the current matching conditions, however, is top limited in terms of power matching conditions. Additionally, and specifically to this method using tunable LEDs as light sources complementing the sun simulator spectrum, the method can be used to identify the AM1.5g performance of a tandem solar cell under a noncalibrated illumination spectrum. A preliminary report on the method has been published earlier [9].

\section{Experimental Details}

Figure 2 shows the experimental setup consisting of a sun simulator with two additional LED sources (with emissions at wavelengths $\lambda \sim 450 \mathrm{~nm}$ and $850 \mathrm{~nm}$, resp.) to tune the spectrum applied to the device under test. The sample is homogeneously illuminated by a sun simulator acting as the wide spectral light source and the LEDs.

The method comprises the following steps: a tandem cell with known quantum efficiency is exposed to the sun simulator spectrum supplemented by different fractions of infrared and blue LED light. Under each illumination condition, an $I V$ curve and the illumination spectra are acquired. In the second step, by acquiring data for another test tandem cell the previously characterized tandem cell can be used as a reference so that the acquisition of the spectra becomes obsolete.

Figure 3(a) displays the external quantum efficiencies (EQE) of the top and bottom cells of an a-Si:H/ $\mu \mathrm{c}$ $\mathrm{Si}: \mathrm{H}$ tandem solar cell. The sample under investigation is deposited on an Asahi U-type substrate and consists of two $p$ - $i-n$ junctions followed by a TCO silver rear reflector layer [10]. Figure 3(b) shows the spectral power densities 


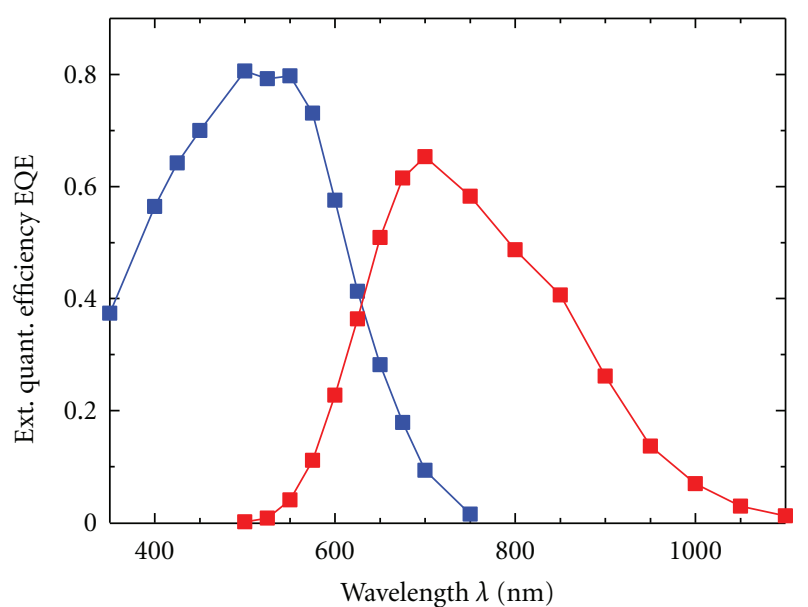

(a)

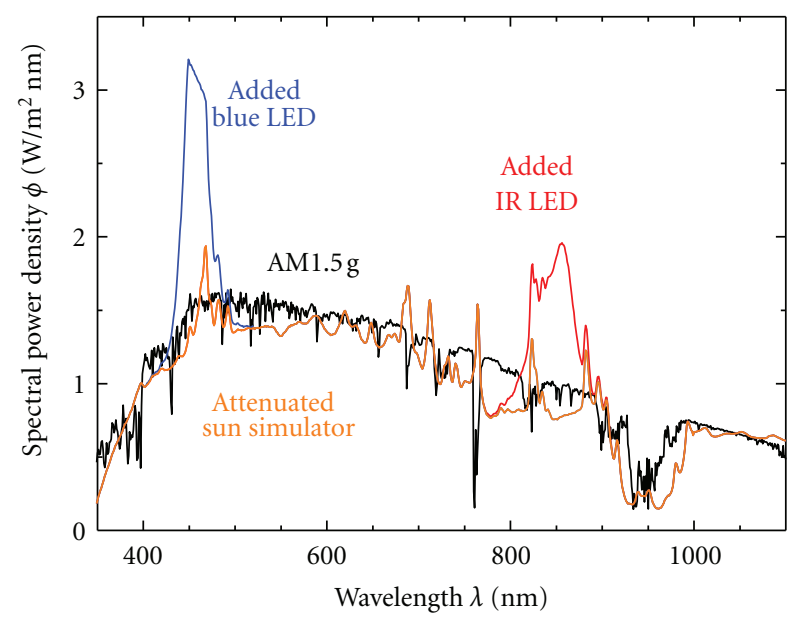

(b)

FIGURE 3: (a) External quantum efficiency EQE of the top (blue) and bottom (red) cell of the tandem solar cell sample. (b) Illuminated power density. The AM1.5g standard is given in black, the attenuated sun simulator spectrum in orange, and the spectra of the full LED illumination in either blue or infrared is given in blue and red, respectively. The LED sources are chosen to affect, respectively, one of the subcells.

corresponding to the AM1.5g spectrum [11], to the spectrum of the sun simulator, and to illumination by the sun simulator with additional light bias provided by the two LED sources, respectively. The total thickness of the tandem cell is $2.4 \mu \mathrm{m}$. It is seen from Figures 3(a) and 3(b) that the wavelengths range of the LED light sources is chosen to affect either the top or the bottom cell exclusively. Furthermore, the output power of the sun simulator is slightly attenuated in order to keep the total light power incident on the cell equal to the standard value of $1000 \mathrm{~W} / \mathrm{m}^{2}$ when the LEDs are added. Therefor the power of the sun simulator light sources was reduced (filters could have been used as well). The LED bias illumination intensity is modified from $100 \%$ infrared (IR) to $100 \%$ blue in equidistant power steps, increasing the power at the IR sources while decreasing the blue sources. The total illumination intensity is kept constant.

\section{Calibration of the Reference Cell}

The experiments described in the following provide a detailed analysis of the matching behavior of an a-Si:H/ $\mu \mathrm{c}-\mathrm{Si}: \mathrm{H}$ tandem solar cell. These experiments include the measurement of the EQE of the top and bottom cells, the measurement of the JV characteristics of this cell under systematically varied contributions from the LEDs, and the measurement of the combined illumination spectra corresponding to each of those measurements. This somewhat cumbersome procedure provides an analysis method on its own right but is especially valuable when using the analyzed cell as a reference cell for a simplified analysis of further cells as described in Section 4.

In the first step, we measure the EQE of the top and the bottom cells of the reference sample (see Figure 3(a)). The second step comprises number $N+1$ of $J V$ measurements $(n=1,2, \ldots, N+1)$ under systematically varied light bias by the LEDs. Here the power provided by each LED is varied by equidistant steps $\Delta \phi_{\mathrm{LED}}^{1}(\lambda)$ and $\Delta \phi_{\mathrm{LED}}^{2}(\lambda)$, for LED 1 and 2 , respectively. At each step the output of LED 1 is increased whereas that of LED 2 is decreased such that we have for the total light intensity arriving at the sample

$$
\begin{aligned}
\phi(n, \lambda)= & (n-1) \Delta \phi_{\mathrm{LED}}^{1}(\lambda)+(N+1-n) \Delta \phi_{\mathrm{LED}}^{2}(\lambda) \\
& +\phi_{\text {sunsim }}(\lambda) .
\end{aligned}
$$

Note that we have tuned the illumination steps $\Delta \phi_{\mathrm{LED}}^{1}(\lambda)$, $\Delta \phi_{\mathrm{LED}}^{2}(\lambda)$, and the output $\phi_{\text {sunsim }}(\lambda)$ of the sun simulator in a way such that the total illumination intensity corresponds to approximately $1000 \mathrm{~W} / \mathrm{m}^{2}$ for each measurement. The original sun simulator spectrum was attenuated to meet the requirement. Yet, experiments with a slightly changed, in terms of spectral shifts, sun simulator spectrum lead to the same results (data not shown).

By using the measured EQEs and the recorded spectra $\phi(n, \lambda)$ we calculate the short-circuit current densities

$$
J_{\mathrm{SC}}^{\mathrm{top} / \mathrm{bot}}(n)=\frac{q}{h c} \int \mathrm{EQE}_{\text {top } / \text { bot }}(\lambda) \phi(n, \lambda) \lambda \mathrm{d} \lambda
$$

for each measurement $n$. In (2), $q$ is the elementary charge, $h$ denotes Planck's constant, $c$ is the velocity of light, $\lambda$ is the wavelength, and $\mathrm{EQE}_{\text {top/bot }}(\lambda)$ are the external quantum efficiencies as given in Figure 3(a). For our measurement setup the uncertainty of $J_{\mathrm{SC}}^{\text {top }}$ and $J_{\mathrm{SC}}^{\text {bot }}$ calculated from their $\mathrm{EQE}$ is $4 \%$. Next we quantify the current mismatch $\Delta J_{\mathrm{SC}}=$ $J_{\mathrm{SC}}^{\text {top }}-J_{\mathrm{SC}}^{\text {bot }}$ by the difference between the current densities of the top and the bottom cells. Since the linear variations of the LED illumination affect only the top cell (LED 1) or the bottom cell (LED 2), we have the linear relationship

$$
\begin{aligned}
\Delta J_{\mathrm{SC}}(n)= & a(n-1) \Delta \phi_{\mathrm{LED}}^{1}(\lambda) \\
& -b(N+1-n) \Delta \phi_{\mathrm{LED}}^{2}(\lambda)+\Delta J_{\mathrm{SC}}^{0} \\
= & (n-1) k_{1}+k_{0} .
\end{aligned}
$$

Here $a, b, k_{0}$, and $k_{1}$ are coefficients describing the relationship mathematically. 


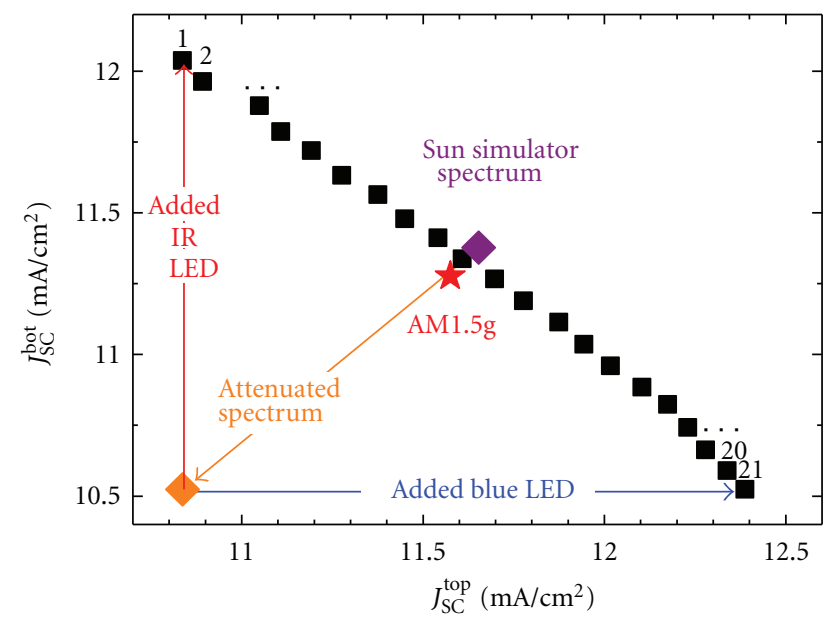

FIGURE 4: The measured external quantum efficiency of the reference cell and the illumination spectra are used to calculate the shortcircuit current densities $J_{\mathrm{SC}}^{\text {top }}$ and $J_{\mathrm{SC}}^{\mathrm{bot}}$ of the subcells. Additionally, the current densities for the AM1.5g standard spectrum (red star) and for the sun simulator spectrum (violet diamond) are given along with the value for the attenuated sun simulator spectrum (orange diamond). The stepwise changed LED illumination (measurements number 1 to 21) leads to, respectively, increased top and decreased bottom cell current densities (black squares).

Figure 4 depicts the variations of the short-circuit current densities $J_{\mathrm{SC}}^{\text {top }}$ and $J_{\mathrm{SC}}^{\text {bot }}$ resulting from the $N+1=21$ measurements using linear spectral variations of the applied illumination. For clarity, the short-circuit current densities calculated for the standard AM1.5g spectrum as well as for the attenuated and not attenuated sun simulator as calculated with the help of (2) are also shown. It can be seen from Figure 4 that by variation of the LED light bias we are able to approximate very well the short-circuit current densities defined by the AM1.5g spectrum as well as those defined by the unattenuated sun simulator. It is worth noting that the present method enables one to analyze the cell close to standard conditions even if the sun simulator is not as well matched to the AM1.5g spectrum as in the present case.

Figure 5 shows the current density difference $\Delta J_{\mathrm{SC}}=$ $J_{\mathrm{SC}}^{\text {top }}-J_{\mathrm{SC}}^{\text {bot }}$ as a function of the index $n$ of the measurement and demonstrates that the linear relationship between $\Delta J_{\mathrm{SC}}$ and $n$ as postulated by (3) is very well fulfilled.

The next step requires the measurement of the $J V$ characteristics of the cell under each of the $N+1$ illumination bias conditions. For each of the curves we determine the maximum output power $P$, the fill factor FF, and the shortcircuit current density $J_{\mathrm{SC}}$. For our measurement setup, the uncertainties for those quantities are roughly $4 \%$. A normalized set of these data as a function of the measurement index $n$ is shown in Figure 6. Note that due to the linear relation between $n$ and the short-circuit current density difference $\Delta J_{\mathrm{SC}}$, we may use $\Delta J_{\mathrm{SC}}$ (instead of $n$ ) as a scale for the display of the data (top axis in Figure 6). As expected, the maximum of the overall short-circuit current density $J_{\mathrm{SC}}$ and the minimum of the fill factor FF is close to $\Delta J_{\mathrm{SC}}=0$, that is, at the current matching condition.

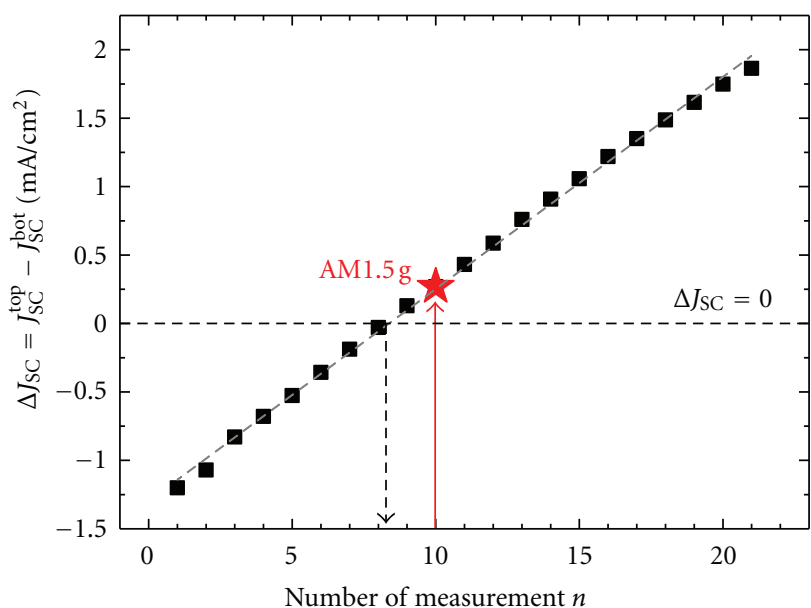

FIGURE 5: The current density difference $\Delta J_{\mathrm{SC}}=J_{\mathrm{SC}}^{\text {top }}-J_{\mathrm{SC}}^{\text {bot }}$ rises linearly as a function of the index $n$ of the measurement as postulated by (3). The gray dashed line is a linear fit. The AM1.5g-illumination conditions are most closely met for measurement number $n=10$, marked in red. The subcell currents are equal, $\Delta J_{\mathrm{SC}}=0 \mathrm{~mA} / \mathrm{cm}^{2}$, approximately for measurement number $n=8$.

$$
\Delta J_{\mathrm{SC}}=J_{\mathrm{SC}}^{\mathrm{top}}-J_{\mathrm{SC}}^{\mathrm{bot}}\left(\mathrm{mA} / \mathrm{cm}^{2}\right)
$$

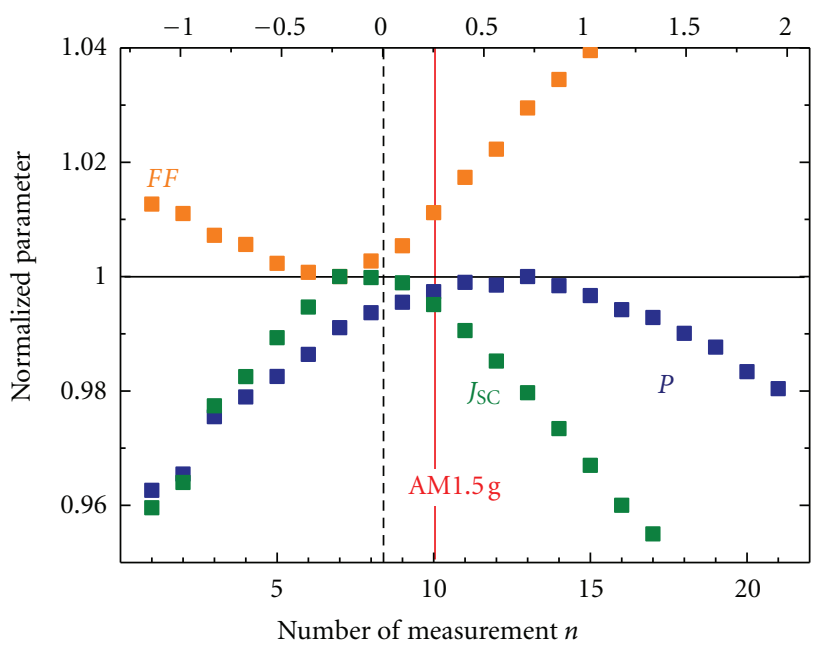

FIGURE 6: The normalized solar cell parameters are given for each measurement number. As the measurement number $n$ is linearly related to the difference in short-circuit current $\Delta J_{\mathrm{SC}}$, the second scale is attached above. The sample is slightly mismatched. The total short-circuit current density $J_{\mathrm{SC}}$ (green symbols) is maximized when the subcell currents are approximately equal, close to $\Delta J_{\mathrm{SC}}=$ 0 , which does not correspond to AM1.5g-illumination conditions. Under the same current matching condition the fill factor (orange symbols) is minimal. The power (blue symbols) peaks for larger values of $\Delta J_{\mathrm{SC}}$. A layer thickness adaption, specifically an increase of the top cell layer thickness, would result in a power increase.

The reference point given by the AM1.5g-standard spectrum is found at $\Delta J_{\mathrm{SC}}(\mathrm{AM} 1.5 \mathrm{~g})=0.25 \mathrm{mAcm}^{-2}$. Thus, we have $J_{\mathrm{SC}}^{\text {top }}>J_{\mathrm{SC}}^{\text {bot }}$ and the cell is bottom limited in terms of the current matching conditions. However, the maximum of the output power is found at $\Delta J_{\mathrm{SC}}\left(P_{\max }\right)=0.72 \mathrm{mAcm}^{-2}$, that is, $\Delta J_{\mathrm{SC}}\left(P_{\max }\right)>\Delta J_{\mathrm{SC}}(\mathrm{AM} 1.5 \mathrm{~g})$ and therefore the cell 
is top limited in terms of power matching conditions. The loss induced by the power mismatch under current matching is about $0.9 \%$ of the power achieved under optimum power matching conditions. However, the value of $\Delta J_{\mathrm{SC}}\left(P_{\max }\right)=$ $0.72 \mathrm{mAcm}^{-2}$ shows that the different matching conditions are met by considerably different situations and lead to considerably different conclusions. The increase in the fill factor of the $J V$ curve partially compensates current losses due to the mismatch. In order to optimize the present cell design for current matching a thicker bottom cell would be needed, whereas optimization of the power matching (and output power) requires a thicker top cell.

\section{Measurements Using the Calibrated Tandem Cell as a Reference}

The full set of experiments described in the previous section is rather time consuming and not suitable for the investigation of many tandem solar cells. In the following, we describe a procedure that does not require the measurement of the EQE of each device nor the determination of the irradiation spectra. The spectrum delivered by the sun simulator does not need to be identical to the calibration measurement but should be unaltered during one test series.

In the following we use four terms describing the different settings: a reference tandem cell (ref, we use the tandem cell and the data that was presented above) and a test tandem cell (test) are both exposed to a calibration illumination (cal, as was done before) and to an experimental test illumination (exp). As for the calibration procedure, we need a series of $J V$ measurements under varied illumination conditions. However, with our automated setup such a measurement series is performed within a few minutes for one sample. We have to perform this measurement under the same conditions for the devices under test as well as for the calibrated reference sample. We apply the method to a top limited (for current as well as power matching) test tandem cell and show only 11 measurement points for the experiment instead of 21 to emphasize the fact that it is not necessary to perform such a large number of measurements in the experiment. Note that the reference sample must not be degraded since the calibration measurement. For the evaluation, we have further to assume that the linearity expressed by (1) and (3) is valid for the test series.

Figure 7 shows the recorded output power for the device under test as well as for the calibrated reference sample as a function of the measurement index of the test procedure (open symbols). Also shown is the output power $P$ of the calibrated reference sample as it was recorded during the calibration measurement (full symbols). Due to (intentional) change of the sun simulator spectrum, the absolute value and the position of the maxima of the two curves for the reference sample are changed. However, due to the linearity in (1) and (3), we are allowed to rescale the $n$ axis of the test measurement by a linear transformation into the $\Delta J_{\mathrm{SC}}^{\mathrm{cal}}$ axis of the calibration measurement according to

$$
\Delta J_{\mathrm{SC}}^{\mathrm{cal}}(n)=c_{1}(n-1)+c_{0}
$$

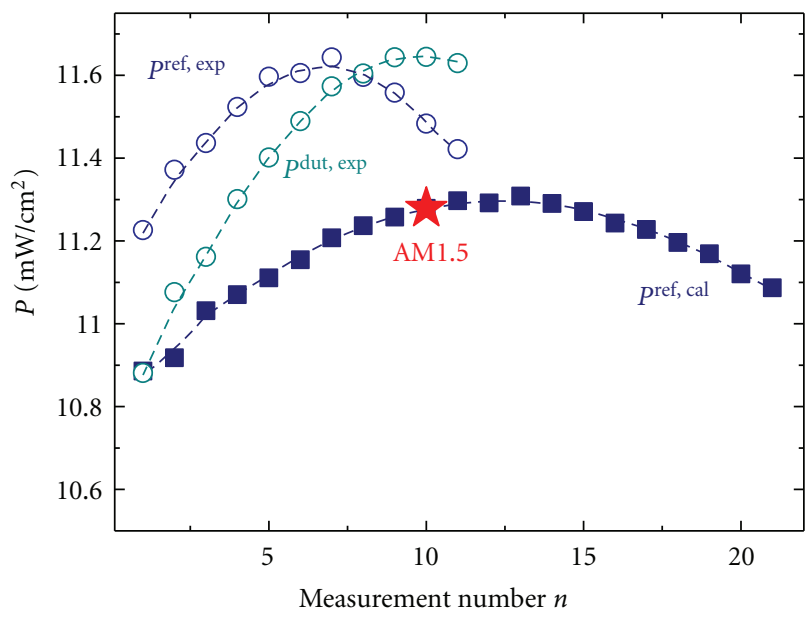

FIgURE 7: The converted power was determined in $J V$ measurements under the sun simulator illumination applying additional LED illumination. The reference tandem solar cell was measured under an attenuated sun simulator spectrum (filled blue symbols) and under a noncalibrated illumination (open blue symbols). The device under test was characterized under the same test spectra (open cyan symbols). The measurement number $n$ labels the different steps in additional LED bias illumination. We show 11 steps for the experiment, 21 in the calibration. The red star marks the illumination conditions closest to AM1.5g.

Figure 8 compares the normalized output parameters, maximum output power $P$, fill factor $\mathrm{FF}$, and short-circuit current density $J_{\mathrm{SC}}$ as obtained from the calibration measurement (same data as in Figure 6, full symbols) to those data obtained during the test measurement after rescaling the $n$ axis of the test measurement to the $\Delta J_{\mathrm{SC}}^{\text {ref, cal }}$ axis of the calibration measurement. The coefficients $c_{1}$ and $c_{0}$ in (4) are chosen to achieve an optimum match between the respective $P$ data. As can be seen from Figure 8, the linear transformation of the $n$ axis into the $\Delta J_{\mathrm{SC}}^{\mathrm{ref} \text { cal }}$ axis reproduces the curves from the calibration measurement very well. The normalized parameters measured under the experimental test illumination and then shifted differ by less than $0.1 \%$ from those measured under calibration conditions.

In the next step, we use the same transformation that was used to recalculate the values of the calibrated reference tandem solar cell under reference illumination conditions. We apply this transformation to the values measured for the test tandem sample under test illumination.

Figure 9 shows the normalized parameters $P, F F$, and $J_{\mathrm{SC}}$ for the test tandem using the scale of the reference sample. The scale of the reference sample under calibration illumination differs from the short-circuit current density difference of the subcells of the test tandem sample measured under test illumination. As axis the short-circuit current difference of the subcell currents of the reference solar cell under calibration illumination is used. The $\Delta J_{S C}^{\text {ref, cal }}$ scale corresponds to a difference of the subcell short-circuit currents $\Delta J_{\mathrm{SC}}^{\text {dut, cal }}$ of the device under test in relative units. At the $\Delta J_{\mathrm{SC}}^{\mathrm{ref}, \mathrm{cal}}$ position of the FF minimum and the $J_{\mathrm{SC}}$ maximum, 


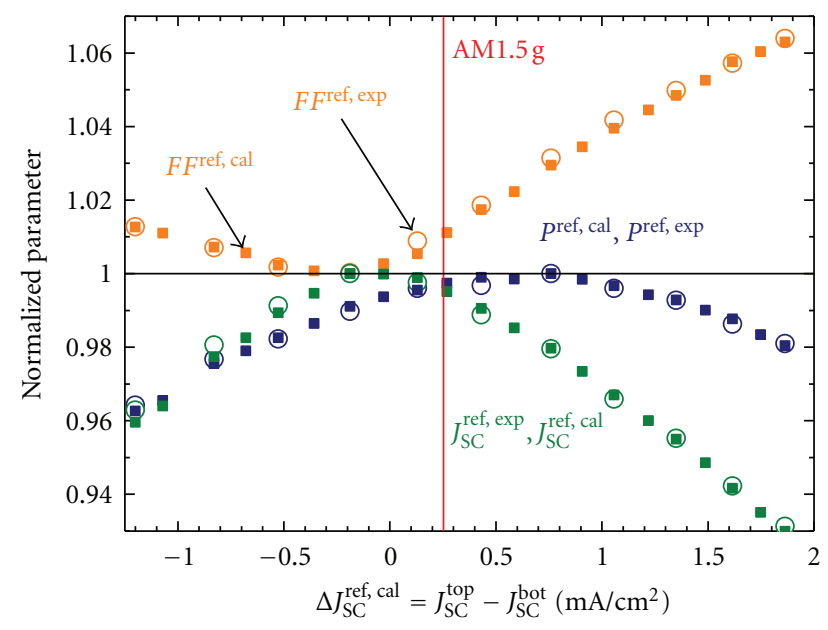

FIGURE 8: The normalized output parameters, maximum output power $P$, fill factor $\mathrm{FF}$, and short-circuit current density $J_{\mathrm{SC}}$ are given as determined for the reference tandem under calibration illumination (ref, cal, same data as Figure 6, filled symbols). The same data acquired under the experimental test illumination was shifted to match the data under calibration conditions (ref, exp, open symbols). The data overlap. The $x$ axis is the one from the calibration measurement.

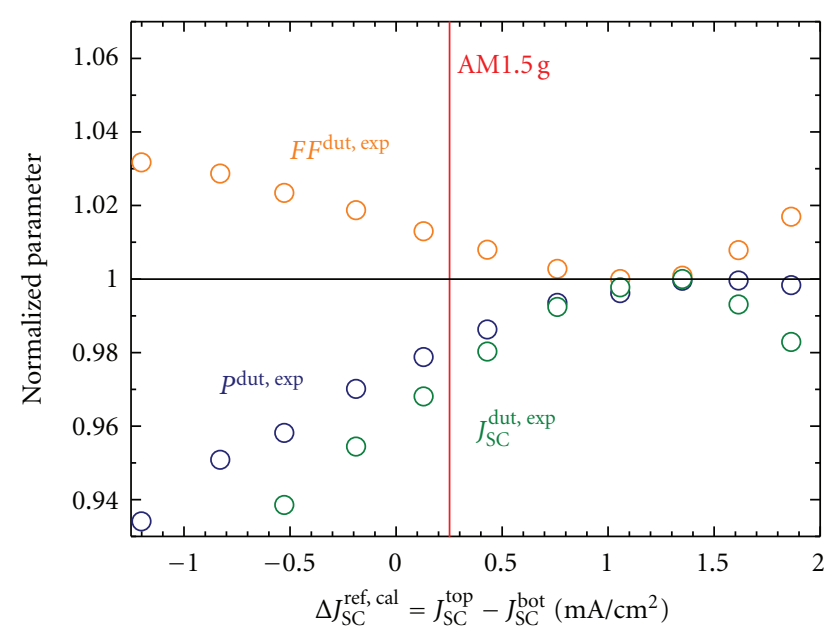

FIGURE 9: The transformation calculated for the calibrated reference is applied to shift the normalized power $P$, fill factor FF, and short circuit current density $J_{S C}$ of the test cell under experimental test illumination (dut, exp) to the calibration illumination conditions. For this test cell power matching and current matching coincide. The device is top limited under AM1.5g illumination.

the test cell is current matched. The red line marks the shortcircuit current difference of the reference for illumination conditions closest to AM1.5g. This illumination corresponds also for the DUT to a current generation as under AM1.5g illumination if the DUT has a quantum efficiency similar to that of the reference at the LED wavelengths. Assuming this was true, we can state that the test cell is top limited under AM1.5g. For a maximum output power, the thickness of the

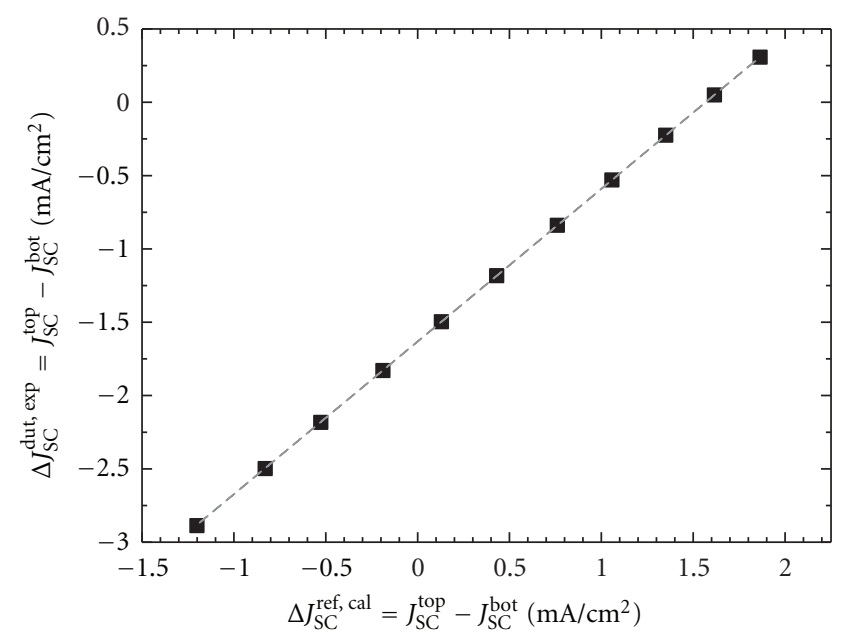

FIGURE 10: With an additional measurement of the external quantum efficiency of the test cell and acquisition of the experimental test spectra, the short-circuit current of the subcells of the test tandem sample under test illumination (dut, exp) can be calculated. The relationship of the differences $\Delta J_{\mathrm{SC}}^{\mathrm{dut}, \exp }$ to the differences $\Delta J_{\mathrm{SC}}^{\mathrm{ref}, \mathrm{cal}}$ of the short-circuit currents for the reference under calibration illumination is linear.

top subcell would have to be increased implying an expected increase in power by $\sim 2 \%$.

Note that in the specific case of this tandem solar cell, the power peaks close to short-circuit current matching. This is due to the good fill factors of the two subcells and implies that current and power matching are very close.

Note also that the proposed method is also interesting for the investigation of multijunction concentrator solar cells, where the fill factor can be a valuable diagnostic tool [12] and (for all types of cells) in the optimization to specific spectra.

\section{Control Experiment}

Figure 10 proves the linear relationship between the current density differences of the device under test under the experimental test illumination $\Delta J_{\mathrm{SC}}^{\mathrm{dut} \text { exp }}$ and of the calibrated reference tandem solar cell under calibration illumination $\Delta J_{\mathrm{SC}}^{\mathrm{ref}, \mathrm{cal}}$. The difference in the current values of the $x$ and $y$ axes comes from the difference in the respective experimental measurement spectra and quantum efficiencies of the subcells of the two tandems. If the DUT and the reference had the same quantum efficiencies in the two wavelength ranges of the LEDs used in the characterization method, the slope of the line would be one. A steeper or shallower slope reflects the difference in the absorption and charge carrier generation in the single subcells with the LED illumination. Keeping this in mind, it is clear that the quantum efficiency of the subcells of the reference tandem cell and of the DUT must meet the sole criterion of being sufficiently high and exclusive (only in one subcell) in the wavelength range of the LEDs.

To prove the concept we also exposed the device under test to reference illumination conditions. Data is shown in Figure 11, full symbols. The shifted values from the 


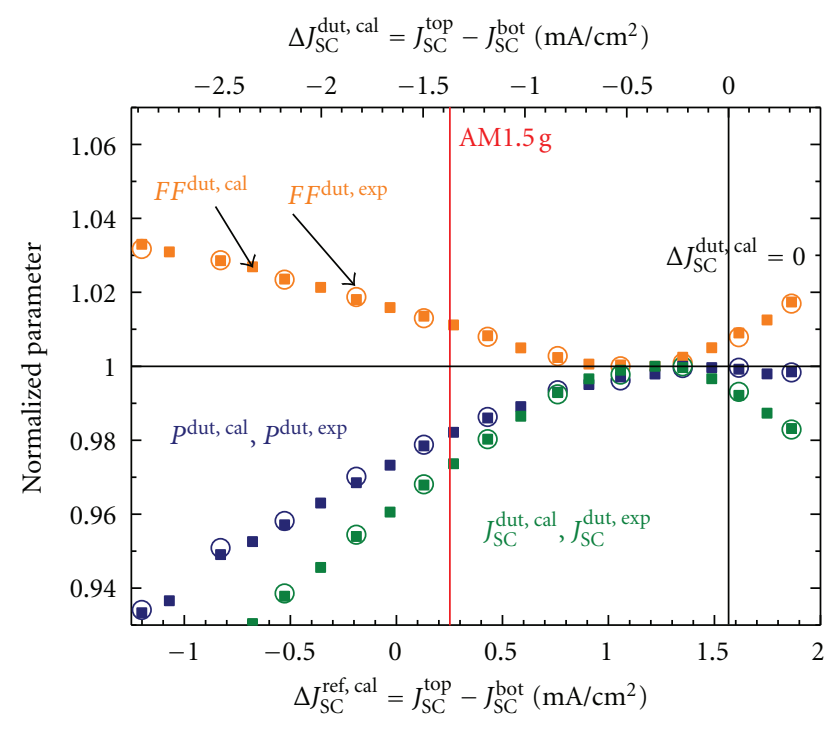

Figure 11: To prove the method, measured data of the tandem device under test under calibration illumination conditions are given (full symbols). The calculated transformation for the experimental test illumination to the calibration conditions is given by open symbols (same data as Figure 9). The fact that the peak position of $J_{\mathrm{SC}}$ differs from $\Delta J_{\mathrm{SC}}^{\text {dut, cal }}=0$ is probably due to measurement uncertainties in the external quantum efficiency.

experiment are reproduced very well. The peak in $J_{\mathrm{SC}}$ is not found at $\Delta J_{\mathrm{SC}}^{\mathrm{dut} \text {, cal }}=0$. The difference is probably due to uncertainties in the external quantum efficiency that was measured with a filter wheel device.

\section{Conclusions}

The proposed characterization tool allows for the quantification of the power mismatch of tandem solar cells and is thus very helpful in the optimization. The power matching of the currents is applied to the maximum performance output instead of the common current matching under short-circuit conditions. The LED-based method additionally enables the determination of the performance under AM1.5g illumination without time-consuming calibration of the sun simulator spectrum. The $J V$ curves measured under IR and blue rich illumination can also be of use in an analysis of the subcell fill factors.

\section{Acknowledgments}

The present work was supported by NRW Ziel2 (project InnoPV, AZ 64.65.69-EN-1022B). The authors thank Thomas Zimmermann for providing the solar cell samples and Bart Pieters for a critical reading of this paper.

\section{References}

[1] J. Nelson, The Physics of the Solar Cell, Imperial College Press, 2003.
[2] J. Burdick and T. Glatfelter, "Spectral response and I-V measurements of tandem amorphous-silicon alloy solar cells," Solar Cells, vol. 18, no. 3-4, pp. 301-314, 1986.

[3] M. Bliss, T. R. Betts, and R. Gottschalg, "Indoor measurement of photovoltaic device characteristics at varying irradiance, temperature and spectrum for energy rating," Measurement Science and Technology, vol. 21, no. 11, Article ID 115701, 2010.

[4] R. Adelhelm and K. Bücher, "Performance and parameter analysis of tandem solar cells using measurements at multiple spectral conditions," Solar Energy Materials and Solar Cells, vol. 50, no. 1-4, pp. 185-195, 1998.

[5] Y. Tsuno, Y. Hishikawa, and K. Kurokawa, "Separation of the IV curve of each component cell of multi-junction solar cells," in Proceedings of the 31st IEEE Photovoltaic Specialists Conference, pp. 1476-1479, January 2005.

[6] M. Meusel, R. Adelhelm, F. Dimroth, A. W. Bett, and W. Warta, "Spectral mismatch correction and spectrometric characterization of monolithic III-V multi-junction solar cells," Progress in Photovoltaics, vol. 10, no. 4, pp. 243-255, 2002.

[7] S. R. Kurtz, K. Emery, and J. M. Olson, "Methods for analysis of two-junction, two-terminal photovoltaic devices," in Proceedings of the 24th IEEE Photovoltaic Specialists Conference, pp. 1733-1737, December 1994.

[8] T. Repmann, J. Kirchhoff, W. Reetz, F. Birmans, J. Müller, and B. Rech, "Investigations on the current matching of highly efficient tandem solar cells based on amorphous and microcrystalline silicon," in Proceddings of the 3rd World Conference on Photovoltaic Energy Conversion, pp. 1843-1846, Osaka, Japan, May 2003.

[9] C. Ulbrich, C. Zahren, J. Noll et al., "Power matching of tandem solar cells," in Proceedings of 26th European Photovoltaic Solar Energy Conference, pp. 302-305, 2011.

[10] T. Merdzhanova, T. Zimmermann, U. Zastrow, W. Beyer, F. Finger, and A. Gordijn, "Single-chamber process for silicon tandem solar cells: role of phosphorous and boron in the tunnel recombination junction," submitted to Journal of Physics D.

[11] IEC, "Photovoltaic devices-Part 3: Measurement principles for terrestrial photovoltaic (PV) solar devices with reference spectral irradiance data," 60904-3 ed. 2, 2008.

[12] W. E. McMahon, K. E. Emery, D. J. Friedman et al., "Fill factor as a probe of current-matching for GaInP2/GaAs tandem cells in a concentrator system during outdoor operation," Progress in Photovoltaics, vol. 16, no. 3, pp. 213-224, 2008. 

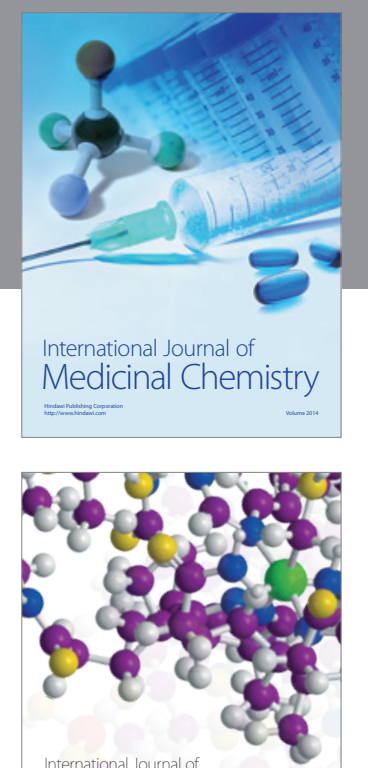

\section{Carbohydrate} Chemistry

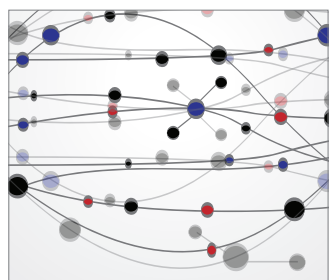

The Scientific World Journal
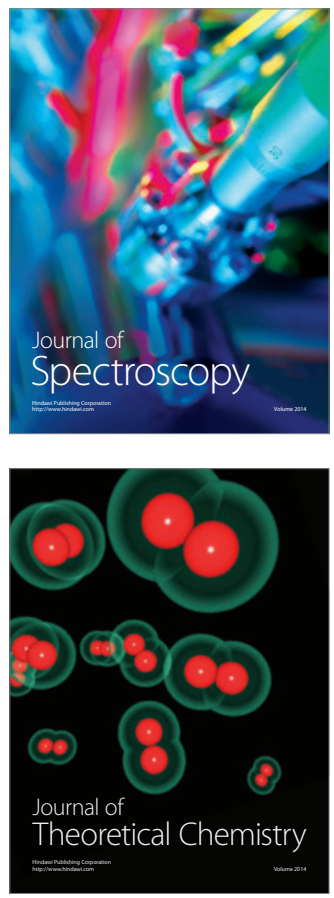
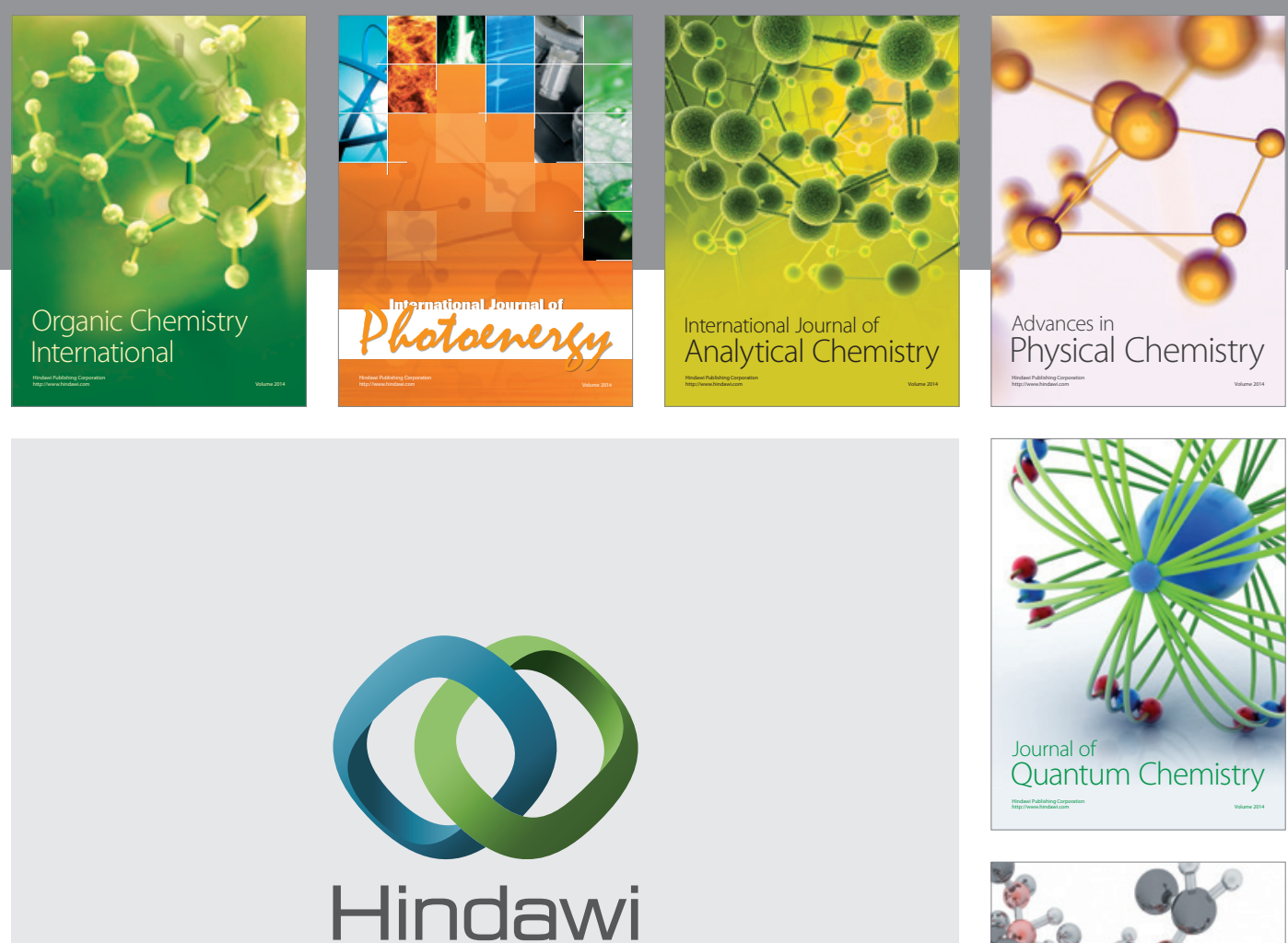

Submit your manuscripts at

http://www.hindawi.com

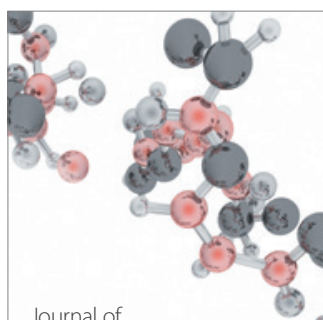

Analytical Methods

in Chemistry

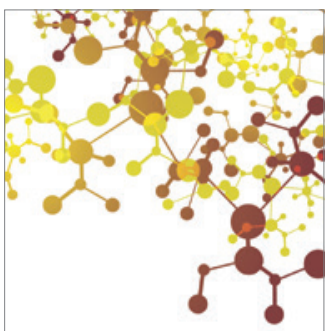

Journal of

Applied Chemistry

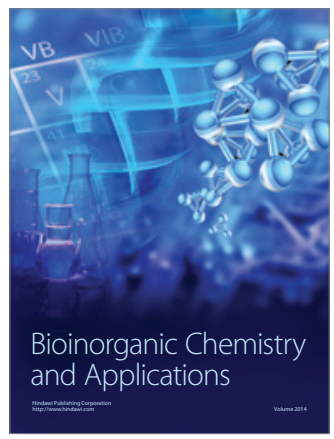

Inorganic Chemistry
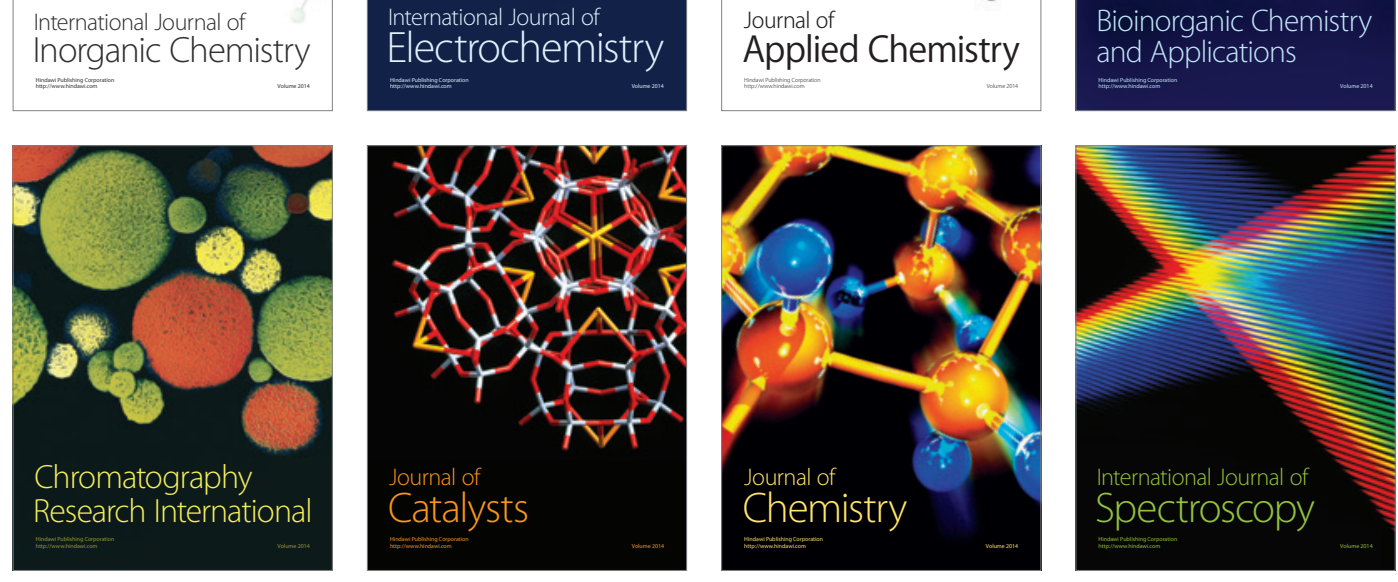